\title{
Challenges in the research and development of new human vaccines
}

\author{
T. Barbosa and M. Barral-Netto
}

Centro de Pesquisas Gonçalo Moniz, Fundação Oswaldo Cruz, Salvador, BA, Brasil

\begin{abstract}
The field of vaccinology was born from the observations by the fathers of vaccination, Edward Jenner and Louis Pasteur, that a permanent, positive change in the way our bodies respond to life-threatening infectious diseases can be obtained by specific challenge with the inactivated infectious agent performed in a controlled manner, avoiding the development of clinical disease upon exposure to the virulent pathogen. Many of the vaccines still in use today were developed on an empirical basis, essentially following the paradigm established by Pasteur, "isolate, inactivate, and inject" the disease-causing microorganism, and are capable of eliciting uniform, long-term immune memory responses that constitute the key to their proven efficacy. However, vaccines for pathogens considered as priority targets of public health concern are still lacking. The literature tends to focus more often on vaccine research problems associated with specific pathogens, but it is increasingly clear that there are common bottlenecks in vaccine research, which need to be solved in order to advance the development of the field as a whole. As part of a group of articles, the objective of the present report is to pinpoint these bottlenecks, exploring the literature for common problems and solutions in vaccine research applied to different situations. Our goal is to stimulate brainstorming among specialists of different fields related to vaccine research and development. Here, we briefly summarize the topics we intend to deal with in this discussion.
\end{abstract}

Key words: Human vaccines; Vaccine issues; Vaccine efficacy; Immunization strategies

Vaccination is one of the most important cost-effective measures for controlling and preventing infectious diseases, being responsible for preventing millions of deaths worldwide every year in spite of suboptimal coverage (1). It is based on the pioneering trials of vaccination performed by Edward Jenner and Louis Pasteur. Since these early efforts, vaccines have been developed against important diseases that have afflicted humanity. The eradication of smallpox, which was considered to be the worst plague of humankind, announced in 1977 by the World Health Organization (WHO), is perhaps the best illustration of the power of this approach. Diseases such as measles and poliomyelitis have been banished from the Americas and there is an effort by the WHO to reach worldwide eradication (2). Vaccinology has emerged as a field that seeks to advance processes and techniques that can more suitably provide the best candidates for rationally designed new vaccines. Even though the number of children's deaths has declined dramatically as vaccination has become more widespread, millions of infants worldwide continue to die every year due to vaccine-preventable diseases (1).

Vaccines were initially developed on an empirical basis, essentially following the paradigm established by
Pasteur, "isolate, inactivate, and inject" the diseasecausing microorganism (3). Such model of vaccine development has crossed the past century and has yielded vaccines basically relying on the attenuation or the inactivation of pathogens (4). The success of this approach has encouraged its massive application to obtain "a vaccine for each disease or pathogen", against all types of infectious diseases.

Unfortunately, although Pasteur's principle has proven to be efficient for developing vaccines against several diseases, such as smallpox, measles and poliomyelitis, numerous other human infectious diseases of diverse etiologies remained without prevention or control using this strategy. The objective of the present group of review articles is to discuss bottlenecks that hinder vaccine development against many diseases, thereby encouraging the debate of these issues by specialists regardless of their focus on a specific pathogen or disease.

The challenges to Pasteur's linear vaccine development strategy "isolate, inactivate, and inject" have appeared early (Figure 1). It soon became clear that for many pathogens the Pasteur principle is not easily applicable. Some disease-causing microorganisms are still not cultivable, such as Mycobacterium leprae (5). 


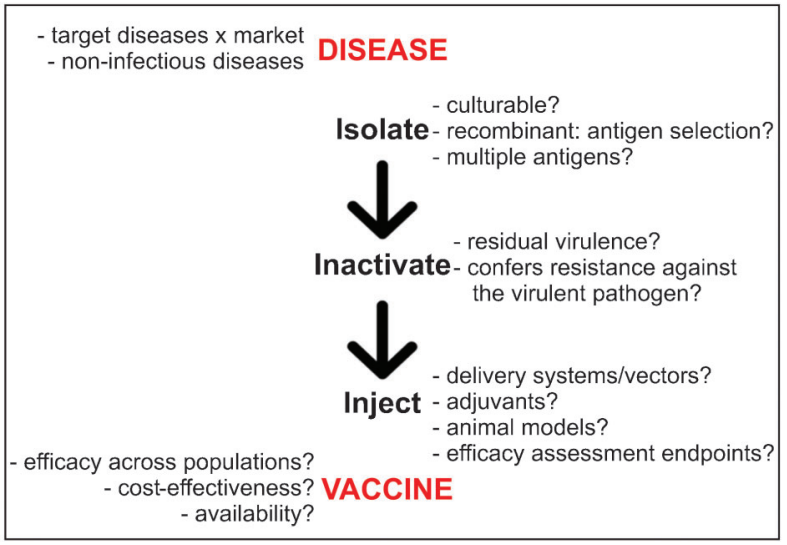

Figure 1. Issues regarding the development of new vaccine strategies. The Pasteur paradigm is highlighted in bold. Issues in vaccine development that are found in the successful accomplishment of each step are highlighted laterally. On the left: some issues of vaccine development are beyond the challenges involved in following the Pasteur paradigm. The choice of the diseases to be targeted by new vaccine strategies may be influenced by aspects that escape the strictly technical issues and challenges imposed by the pathogen: the market expectations of profit, as well as the costs to the end consumer (either to individual subjects or governments), may limit the availability and usefulness of new vaccine strategies to reduce the burden of infectious diseases. On the other hand, non-infectious conditions, as listed in the text, have been addressed as targets for the development of new vaccines, and the strategies for their development are not covered by the Pasteur paradigm. The genetic variation among human populations may influence vaccine efficacy, as already shown for vaccines currently in use, which may raise the need for specific vaccines for different regions of the world. On the right: even for infectious diseases the steps suggested by the Pasteur paradigm may yield important issues to be overcome during vaccine research and development. Not all pathogens are culturable, rendering strategies such as the use of inactivated or attenuated organisms not applicable to some diseases. When selecting pathogen antigens to be obtained without the need to culture the organism, issues regarding which antigen(s) to choose and the antigenic variation of the pathogen will often need consideration. Inactivated organisms may retain residual virulence that may limit their usefulness in susceptible populations, or may lack antigen determinants or adjuvant properties that are needed for optimal stimulation of a proper immune response to the pathogen targeted. An optimal response to antigen administration is influenced by the delivery systems, vectors and adjuvants of choice, which impact on variables such as route of entry and need of multiple doses. Suitable strategies to assess the efficacy of newly proposed vaccine strategies may also need to be developed, from appropriate animal models to adequate markers of protection that may serve as evaluation endpoints other than the frequency of disease in tested populations.

Some attenuated vaccine strains have a residual risk of producing disease $(6,7)$. Furthermore, there is evidence that virulent pathogen strains causing clinical disease may be capable of subverting and evading the host's immune response in spite of proper vaccine elicitation of protec- tion-related immune mechanisms by less virulent vaccine organisms (8).

While component-based vaccines obtained by recombination technologies without the need to culture the pathogenic organism have been successfully obtained (4), with the added advantage of abolishing the risk of residual virulence, the major challenge is still to select the given antigen(s) that will allow the elicitation of a protective immune response to the pathogen in a consistent manner. The approaches to antigen selection have evolved from empirical tests of single components to high throughput screening (9), but in both cases antigen selection is performed on the basis of our often limited understanding of the phenomena involved in host-pathogen interaction, which guides our selection of the properties that are desirable for the successful candidate. Antigen selection also has to take into consideration the intra-species and intra-host antigenic variation of the target pathogen, which may result from its genetic diversity and/or escape mechanisms (10). Noteworthy, as shown for pneumococcus (11) and influenza (12), the replacement of major strains associated with disease with otherwise less represented vaccine-resistant strains after massive vaccination has been shown to occur as a result of vaccine selection pressure that changes the population dynamics of the disease-producing organisms. In the opening review of this group of articles, Lemaire et al. (13) (Table 1) discuss the modern approaches to the selection of candidate antigens, which take into account the genetic variation of both pathogen and host and the knowledge generated within different disciplines about how these entities interact and the spectrum of scenarios this interaction can produce, from resistance to susceptibility to disease.

Unlike live organisms, killed pathogens, inactivation products and particularly isolated pathogen components will often lack the ability of properly eliciting by themselves the innate immune response (14), a step that is required for immune memory induction (15). This is circumvented by adding components that aid with the proper development of the immune responses to the candidate vaccine antigen, which are known as adjuvants (14). Their mechanisms of action are still not fully understood. It is known, however, that adjuvants do not act uniformly on the immune system, and adjuvant development and selection have proven to be of the utmost importance for the success of new vaccine strategies (16). Adjuvant research is the theme of the review by Pérez et al. (17) (Table 1), which highlights the role of adjuvants and formulation in the generation of appropriate immune responses to vaccine antigens, and also raises important issues regarding the licensing of new adjuvants.

The use of recombinant live vectors, consisting of live organisms incapable of inducing disease but preserving the ability to elicit the innate immune system, is still at an early stage: the first human vaccine using a viral vector is 
Table 1. Titles of Challenges in the Research and Development of New Human Vaccines.

Coping with genetic diversity: the contribution of pathogen and human genomics to modern vaccinology

Human prophylactic vaccine adjuvants and their essential role in new vaccine formulations

Recombinant vaccines and the development of new vaccine strategies

Experimental models in vaccine research
Lemaire et al. (13)

Pérez et al. (17)

Nascimento and Leite (19)

Teixeira and Gomes (27) expected to be approved this year (18). The major concerns about using such strategy are: 1) the residual risk of causing disease, especially in immunocompromised or not fully immunocompetent individuals (children, the elderly, immunosuppressed individuals), and 2) the so far unverified possibility of facilitating the transfer of genetic material from the inoculated vaccine organisms to the "wild" organisms that inhabit the bodies of all of us. This genetic material could confer antibiotic or other less predictable resistance to such wild strains. Nascimento and Leite (19) (Table 1) present an overview of recombinant vaccine strategies and their use in different prime-boost schemes, and also highlight the issues still pending regarding their safety for human use.

Returning to the Pasteur paradigm, once the vaccine candidate is isolated and assuming it is fairly incapable of causing disease or important side effects, it will be subjected to proof by being injected and tested. Even though the earliest attempts at vaccine development have indeed involved direct human testing of the vaccine candidates (1), it is clear that prior to this stage both the safety and the potential efficacy of the vaccine candidate need to be proven by using alternative tests. Animals have been used since the earliest vaccine development efforts as surrogates for the assessment of the safety and efficacy of proposed vaccine candidates (20). However, their responses both to pathogens affecting humans and to vaccine candidates may not be consistently similar to what is ultimately observed in humans, as observed for bacterial (21), viral (22), and parasitic (23) chronic infectious diseases. Furthermore, human immune responses to vaccination are influenced by genetic polymorphisms (24), nutritional status (25), infections and other morbidities (26), and by the exposure to environmental organisms (26), factors that are in general kept under control in experimental animals undergoing testing. Addressing such variation is a matter of concern in the translation of pre-clinical results and expectations to clinical and pragmatic clinical trials. Teixeira and Gomes (27) (Table 1) discuss the advantages and concerns regarding the use of animals in vaccine development, with special focus on the development of vaccines against malaria and leishmaniasis.

The primary goal of vaccination is to avoid the establishment of disease following a natural encounter with the pathogen. However, some pathogens of public health concern may infect the host for long, even life-long periods, without ever causing disease. Furthermore, some of these are very successful pathogens, which infect a considerable parcel of the population: the tuberculosis bacilli (28) and cytomegalovirus (29) are examples. For such pathogens it would be desirable to develop vaccines that would be effective even if administered post-exposure. The major challenge to the success of this approach is the pursuit not of the mounting of a proper immune response from zero, but rather of attaining a favorable modulation of the immune response that is already ongoing against that pathogen. This will require a deep understanding of the pathogen's escape mechanisms that prevent its elimination by the immune system on the one hand, and of the host active mechanisms that restrain pathogen multiplication and disease production on the other. It is worth mentioning that sterilizing immunity may also not be the ideal goal to pursue in order to avoid disease establishment, since exacerbated immune responses against pathogen challenge can also lead to severe disease (30).

The assessment of vaccine efficacy can be impaired by the fact that the traditional endpoint represented by pathogen sterilization may not be feasible to obtain, or desirable, or sufficient to restrain pathology. For chronic infections with long pathogen latency periods, with not all infected individuals progressing to disease, the long time frame and large sample size necessary to evaluate vaccine efficacy in field clinical trials also imply that alternative methods to assess vaccine efficacy will need to be employed, in order to consider only very promising candidates for later phases of vaccine assessment. In this respect, the search for surrogates of protection and biomarkers of disease severity has been considered to be a research priority within the vaccinology field (31-33). The validation of these markers is not simple, requiring not only a deep understanding of the host-pathogen interaction and disease progression-related mechanisms integrated in complex regulatory networks $(34,35)$, but also the tackling of issues associated with the translation to humans of the results obtained using animal models (31), as well as dealing with statistical tools capable of addressing the problems of multi-testing, confounding and bias (36) when assessing the relevance of each marker or set of markers. Noteworthy, a TB vaccine blueprint has been recently published highlighting the need for efforts to integrate biological state-of-the-art technologies in order to identify "signatures of efficacy" to evaluate the induction of protective responses as a fundamental strategy for TB research and development (37). 
Finally, this group of review articles also welcomes to this debate the discussion of other issues regarding the use and regulation of newly proposed vaccines. As we gain better understanding of the activities of the immune system both in its homeostasis and during disease, it also becomes possible to use vaccination strategies to prevent and cure non-infectious diseases. Vaccines may not only be applicable against inflammatory, auto-aggressive conditions that derive from a deregulated, dysfunctional immune system, such as inflammatory bowel diseases (38-40), diabetes (41-43) and multiple sclerosis $(44,45)$, but also against cancer $(46,47)$, and degenerative disorders $(48,49)$, and even for contraception $(50,51)$. These novel uses for vaccine strategies constitute a challenge per se to the field of vaccinology, not only because of the conceptual and technological issues that need to be overcome to make them possible, but also because non-infectious diseases constitute an evergrowing problem to be addressed with public health measures (52).

The promising success in the development of new vaccine strategies raises still another challenge, i.e., how to predict and assess the effects of vaccination on untargeted immune responses. Although the possibility of adverse effects to some currently used vaccines has raised political and social concern in many instances (53), which justify more research to guarantee the safety of their administration, the possibility that nonspecific effects on host immunity may be elicited by vaccine administration needs to be further explored (54). Among these issues is the problem of multiple vaccination, especially among children, as they are the target population of many of the immunization strategies both currently used and under development. Vaccine administration schemes

\section{References}

1. Centers for Disease Control and Prevention. Vaccine preventable deaths and the Global Immunization Vision and Strategy, 2006-2015. MMWR Morb Mortal Wkly Rep 2006; 55: 511-515

2. de Quadros CA. Global eradication of poliomyelitis and measles: another quiet revolution. Ann Intern Med 1997; 127: $156-158$

3. Seder RA, Mascola JR. Basic immunology of vaccine development. In: Bloom BB, Lambert P-H (Editors), The vaccine book. Orlando: Academic Press; 2003. p 51-72.

4. Plotkin SA. Vaccines: past, present and future. Nat Med 2005; 11: S5-S11, doi: 10.1038/nm1209.

5. Lahiri R, Randhawa B, Krahenbuhl J. Application of a viability-staining method for Mycobacterium leprae derived from the athymic (nu/nu) mouse foot pad. $J$ Med Microbiol 2005; 54: 235-242, doi: 10.1099/jmm.0.45700-0.

6. Shimizu H, Thorley B, Paladin FJ, Brussen KA, Stambos V, Yuen $\mathrm{L}$, et al. Circulation of type 1 vaccine-derived poliovirus in the Philippines in 2001. J Virol 2004; 78: 13512-13521, doi: 10.1128/JVI.78.24.13512-13521.2004. need to be better studied to help address these issues. Also the effects of the combined stimulation of the organism with multiple antigens and adjuvants on the efficacy of each vaccine against its specific pathogen need to be further explored, as well as the combined adverse effects they may cause when given concomitantly or separated by short intervals.

Some required solutions of vaccinology challenges will also extrapolate the science-technology field. The local validation of results obtained in pragmatic trials will be necessary, since it has already been shown that different populations can vary significantly in their average responses to a given vaccine (55). Countries differ in terms of policies regarding the local validation of efficacy studies. They also have different policies regarding the marketing of new vaccines, which may not be made widely available due to their cost or because of the choice of targeting specific populations (56). Restrictions to vaccine coverage may interfere with the success of vaccination due to a diminished herd effect (57). Given the fact that infectious diseases constitute a burden that can ultimately interfere with the socioeconomic development of a nation (58), vaccine research should be regarded as strategic not only from the point of view of public health.

In summary, the objective of the present group of review articles is to describe the state-of-the-art scientific and technologic resources that allow us to address the obstacles to a more rational design of new human vaccines. We extend this debate to consider the frontier between what is being done within the scientific and technology field and on sociopolitical grounds to help make vaccines available for the current scourges that affect human populations worldwide.

7. Movahedi Z, Norouzi S, Mamishi S, Rezaei N. BCGiosis as a presenting feature of a child with chronic granulomatous disease. Braz J Infect Dis 2011; 15: 83-86, doi: 10.1016/ S1413-8670(11)70146-5.

8. Kremer K, van-der-Werf MJ, Au BK, Anh DD, Kam KM, vanDoorn HR, et al. Vaccine-induced immunity circumvented by typical Mycobacterium tuberculosis Beijing strains. Emerg Infect Dis 2009; 15: 335-339, doi: 10.3201/ eid1502.080795

9. Pajon R, Yero D, Niebla O, Climent Y, Sardinas G, Garcia $D$, et al. Identification of new meningococcal serogroup $B$ surface antigens through a systematic analysis of neisserial genomes. Vaccine 2009; 28: 532-541, doi: 10.1016/j.vaccine.2009.09.128.

10. Kumkhaek C, Phra-Ek K, Renia L, Singhasivanon P, Looareesuwan S, Hirunpetcharat $C$, et al. Are extensive $T$ cell epitope polymorphisms in the Plasmodium falciparum circumsporozoite antigen, a leading sporozoite vaccine candidate, selected by immune pressure? J Immunol 2005; 175: 3935-3939. 
11. Lipsitch M. Bacterial vaccines and serotype replacement: lessons from Haemophilus influenzae and prospects for Streptococcus pneumoniae. Emerg Infect Dis 1999; 5: 336345, doi: 10.3201/eid0503.990304.

12. Iwami S, Takeuchi Y, Liu X, Nakaoka S. A geographical spread of vaccine-resistance in avian influenza epidemics. J Theor Biol 2009; 259: 219-228, doi: 10.1016/j.jtbi.2009.03.040.

13. Lemaire DC, Barbosa $T$, Rihet $P$. Coping with genetic diversity: The contribution of pathogen and human genomics to modern vaccinology. Braz J Med Biol Res 2012; 45: 376-385, doi: 10.1590/S0100-879X2011007500142.

14. McKee AS, Macleod MK, Kappler JW, Marrack P. Immune mechanisms of protection: can adjuvants rise to the challenge? BMC Biol 2010; 8: 37, doi: 10.1186/1741-7007-8-37.

15. Castellino F, Galli G, Del Giudice G, Rappuoli R. Generating memory with vaccination. Eur J Immunol 2009; 39: 21002105, doi: 10.1002/eji.200939550.

16. Coffman RL, Sher A, Seder RA. Vaccine adjuvants: putting innate immunity to work. Immunity 2010; 33: 492-503, doi: 10.1016/j.immuni.2010.10.002.

17. Pérez O, Batista A, González E, Zayas C, Balboa J, Cuello M, et al. Human prophylactic vaccine adjuvants and their essential role in new vaccine formulations. Braz J Med Biol Res 2012; 45: 681-692, doi: 10.1590/S0100-879X2012007500067.

18. Rollier CS, Reyes-Sandoval A, Cottingham MG, Ewer K, Hill AV. Viral vectors as vaccine platforms: deployment in sight. Curr Opin Immunol 2011; 23: 377-382, doi: 10.1016/ j.coi.2011.03.006.

19. Nascimento IP, Leite LC. Recombinant vaccines and the development of new vaccine strategies. Braz J Med Biol Res 2012; 45: 1102-1111, doi: 10.1590/S0100879X2012007500142.

20. Zepp F. Principles of vaccine design-Lessons from nature. Vaccine 2010; 28 (Suppl 3): C14-C24, doi: 10.1016/ j.vaccine.2010.07.020.

21. Gupta UD, Katoch VM. Animal models of tuberculosis for vaccine development. Indian J Med Res 2009; 129: 11-18.

22. Kimura K, Kohara M. An experimental mouse model for hepatitis C virus. Exp Anim 2011; 60: 93-100, doi: 10.1538/ expanim.60.93.

23. Gupta S. Visceral leishmaniasis: experimental models for drug discovery. Indian J Med Res 2011; 133: 27-39.

24. Poland GA, Ovsyannikova IG, Jacobson RM. Vaccine immunogenetics: bedside to bench to population. Vaccine 2008; 26: 6183-6188, doi: 10.1016/j.vaccine.2008.06.057.

25. Moore SE, Jalil F, Ashraf R, Szu SC, Prentice AM, Hanson LA. Birth weight predicts response to vaccination in adults born in an urban slum in Lahore, Pakistan. Am J Clin Nutr 2004; 80: 453-459.

26. de Bruyn G. Cofactors that may influence vaccine responses. Curr Opin HIV AIDS 2010; 5: 404-408, doi: 10.1097/COH.0b013e32833d1fca.

27. Teixeira C, Gomes R. Experimental models in vaccine research. Braz J Med Biol Res 2013; 46: 109-116, doi: 10.1590/1414-431X20122460.

28. Zumla A, Atun R, Maeurer M, Mwaba P, Ma Z, O'Grady J, et al. Viewpoint: Scientific dogmas, paradoxes and mysteries of latent Mycobacterium tuberculosis infection. Trop Med Int Health 2011; 16: 79-83, doi: 10.1111/j.13653156.2010.02665.x.

29. Syggelou A, lacovidou N, Kloudas S, Christoni Z,
Papaevangelou V. Congenital cytomegalovirus infection. Ann N Y Acad Sci 2010; 1205: 144-147, doi: 10.1111/ j.1749-6632.2010.05649.x.

30. Carvalho LP, Passos S, Bacellar O, Lessa M, Almeida RP, Magalhaes $A$, et al. Differential immune regulation of activated $\mathrm{T}$ cells between cutaneous and mucosal leishmaniasis as a model for pathogenesis. Parasite Immunol 2007; 29: 251-258, doi: 10.1111/j.1365-3024.2007.00940.x.

31. Kim JH, Rerks-Ngarm S, Excler JL, Michael NL. HIV vaccines: lessons learned and the way forward. Curr Opin HIV AIDS 2010; 5: 428-434, doi: 10.1097/COH.0b013e32833d17ac.

32. Sasada T, Komatsu N, Suekane S, Yamada A, Noguchi M, Itoh K. Overcoming the hurdles of randomised clinical trials of therapeutic cancer vaccines. Eur J Cancer 2010; 46: 1514-1519, doi: 10.1016/j.ejca.2010.03.013.

33. Parida SK, Kaufmann SH. The quest for biomarkers in tuberculosis. Drug Discov Today 2010; 15: 148-157, doi: 10.1016/j.drudis.2009.10.005.

34. Braga-Neto UM, Marques ET Jr.. From functional genomics to functional immunomics: new challenges, old problems, big rewards. PLoS Comput Biol 2006; 2: e81, doi: 10.1371/ journal.pcbi.0020081.

35. Bucasas KL, Franco LM, Shaw CA, Bray MS, Wells JM, Nino D, et al. Early patterns of gene expression correlate with the humoral immune response to influenza vaccination in humans. J Infect Dis 2011; 203: 921-929, doi: 10.1093/ infdis/jiq156.

36. Dakna M, Harris K, Kalousis A, Carpentier S, Kolch W, Schanstra JP, et al. Addressing the challenge of defining valid proteomic biomarkers and classifiers. BMC Bioinformatics 2010; 11: 594, doi: 10.1186/1471-2105-11-594.

37. Brennan MJ, Thole J. Tuberculosis vaccines: a strategic blueprint for the next decade. Tuberculosis 2012; 92 (Suppl 1): S6-S13, doi: 10.1016/S1472-9792(12)70005-7.

38. Ohkawara T, Koyama Y, Onodera S, Takeda H, Kato M, Asaka $\mathrm{M}$, et al. DNA vaccination targeting macrophage migration inhibitory factor prevents murine experimental colitis. Clin Exp Immunol 2011; 163: 113-122, doi: 10.1111/ j.1365-2249.2010.04277.x.

39. Beatty PL, Narayanan S, Gariepy J, Ranganathan S, Finn OJ. Vaccine against MUC1 antigen expressed in inflammatory bowel disease and cancer lessens colonic inflammation and prevents progression to colitis-associated colon cancer. Cancer Prev Res 2010; 3: 438-446, doi: 10.1158/19406207.CAPR-09-0194.

40. Guan Q, Ma Y, Hillman CL, Ma A, Zhou G, Qing G, et al. Development of recombinant vaccines against IL-12/IL-23 p40 and in vivo evaluation of their effects in the downregulation of intestinal inflammation in murine colitis. Vaccine 2009; 27: 7096-7104, doi: 10.1016/j.vaccine.2009.09.058.

41. Denes B, Fodor I, Langridge WH. Autoantigens plus interleukin-10 suppress diabetes autoimmunity. Diabetes Technol Ther 2010; 12: 649-661, doi: 10.1089/ dia.2009.0116.

42. Santos RR Junior., Sartori A, Lima DS, Souza PR, CoelhoCastelo AA, Bonato VL, et al. DNA vaccine containing the mycobacterial hsp65 gene prevented insulitis in MLD-STZ diabetes. J Immune Based Ther Vaccines 2009; 7: 4, doi: 10.1186/1476-8518-7-4.

43. Ma YJ, Lu Y, Hou J, Dong YK, Du MZ, Xing Y, et al. Vaccination of non-obese diabetic mice with a fragment 
of peptide P277 attenuates insulin-dependent diabetes mellitus. Int Immunopharmacol 2011; 11: 1298-1302, doi: 10.1016/j.intimp.2011.04.012.

44. Zorzella-Pezavento SF, Chiuso-Minicucci F, Franca TG, Ishikawa LL, Martins DR, Silva CL, et al. Immunization with pVAXhsp65 decreases inflammation and modulates immune response in experimental encephalomyelitis. Neuroimmunomodulation 2010; 17: 287-297, doi: 10.1159/ 000292018.

45. Xue X, Feng G, Li M, Qin X, Wu S, Zhang C, et al. Amelioration of experimental autoimmune encephalomyelitis by BLyS autovaccine. Vaccine 2008; 26: 2873-2881, doi: 10.1016/j.vaccine.2008.03.043.

46. Palucka K, Ueno H, Banchereau J. Recent developments in cancer vaccines. J Immunol 2011; 186: 1325-1331, doi: 10.4049/jimmunol.0902539.

47. Klebanoff CA, Acquavella N, Yu Z, Restifo NP. Therapeutic cancer vaccines: are we there yet? Immunol Rev 2011; 239: 27-44, doi: 10.1111/j.1600-065X.2010.00979.x.

48. Hickman DT, Lopez-Deber MP, Ndao DM, Silva AB, Nand $D$, Pihlgren $M$, et al. Sequence-independent control of peptide conformation in liposomal vaccines for targeting protein misfolding diseases. J Biol Chem 2011; 286: 1396613976, doi: 10.1074/jbc.M110.186338.

49. Chackerian B. Virus-like particle based vaccines for Alzheimer disease. Hum Vaccin 2010; 6: 926-930, doi: 10.4161/hv.6.11.12655.

50. Lemons AR, Naz RK. Contraceptive vaccines targeting factors involved in establishment of pregnancy. Am J Reprod Immunol 2011; 66: 13-25, doi: 10.1111/j.1600-0897.2011.01001.x.

51. Purswani S, Talwar GP. Development of a highly immunogenic recombinant candidate vaccine against human chorionic gonadotropin. Vaccine 2011; 29: 2341-2348, doi: 10.1016/ j.vaccine.2010.11.069.

52. Boutayeb A. The double burden of communicable and noncommunicable diseases in developing countries. Trans $R$ Soc Trop Med Hyg 2006; 100: 191-199, doi: 10.1016/ j.trstmh.2005.07.021.

53. Kesselheim A. Safety, supply, and suits - litigation and the vaccine industry. N Engl J Med 2011; 364: 1485-1487, doi: 10.1056/NEJMp1102182.

54. Salemi S, D'Amelio R. Could autoimmunity be induced by vaccination? Int Rev Immunol 2010; 29: 247-269, doi: 10.3109/08830181003746304.

55. Emch M, Ali M, Acosta C, Yunus M, Sack DA, Clemens JD. Efficacy calculation in randomized trials: global or local measures? Health Place 2007; 13: 238-248, doi: 10.1016/ j.healthplace.2006.01.005.

56. Jauregui B, Sinha A, Clark AD, Bolanos BM, Resch S, Toscano CM, et al. Strengthening the technical capacity at country-level to make informed policy decisions on new vaccine introduction: lessons learned by PAHO's ProVac Initiative. Vaccine 2011; 29: 1099-1106, doi: 10.1016/ j.vaccine.2010.11.075.

57. Brisson M, Edmunds WJ. Economic evaluation of vaccination programs: the impact of herd-immunity. Med Decis Making 2003; 23: 76-82, doi: 10.1177/0272989X02239651.

58. Hotez PJ, Molyneux DH, Fenwick A, Ottesen E, Ehrlich SS, Sachs JD. Incorporating a rapid-impact package for neglected tropical diseases with programs for HIV/AIDS, tuberculosis, and malaria. PLoS Med 2006; 3: e102, doi: 10.1371/journal.pmed.0030102. 